\title{
Narrativas innaturales, ciencia ficción y neoliberalismo en Costa Rica
}

\section{Unnatural Narratives, Science Fiction, and Neoliberalism in Costa Rica}

\author{
Roy Alfaro Vargas*
}

\section{RESUMEN}

Este artículo analiza, dentro de los parámetros de los estudios latinoamericanos marxistas de la cultura y los medios, el fenómeno de la ciencia ficción en Costa Rica. En este sentido, este artículo parte de la crítica de las posiciones de Óscar Gerardo Alvarado Vega (2015), con respecto a la denominada ciencia ficción costarricense, con el fin de sintetizar varias nociones que se han desarrollado en la discusión del fenómeno de tal ciencia ficción, en los últimos tiempos, en Costa Rica. De esta manera, se establece un rechazo de la noción de ciencia ficción costarricense, para acceder a una reconceptualización de tal manifestación como parte del desarrollo de las narrativas innaturales en Costa Rica, ligadas a concepciones post-postmodernas y neoliberales ya presentes en la primera fase de la Promoción del 80.

Palabras clave: Estudios latinoamericanos marxistas, post-postmodernismo, literatura costarricense, cine.

RECIBIDO: Mayo 2018

\section{ACEPTADO: Agosto 2018}

\section{ABSTRACT}

This article analyzes, within the parameters of the Latin American Marxist Studies of Culture and Media, the phenomenon of science fiction in Costa Rica. In this way, this article begins with a critique of Óscar Gerardo Alvarado Vega's positions in regard with the so-called Costa Rican science fiction as a means to synthetize several notions that have recently been developed in the discussion of such science fiction in Costa Rica. Thus, it is established a refusal of the notion of Costa Rican science fiction so as to access to a re-conceptualization of such a manifestation as a part of the development of the unnatural narratives in Costa Rica, linked to some post-postmodern and neoliberal conceptions already present in the first phase of the Generation of 80s.

\footnotetext{
* Investigador independiente. ID: 0000-0001-5893-2415 , Costa Rica. royalfarov@yandex.com 468
} 
Keywords: Latin American Marxist Studies, Post-postmodernism, Costa Rican Literature, Movies.

Instead of expressing and reflecting the movement of the content, the dialectic produces this movement. It is not so much a method of analysis as a method of synthetic and systematic construction of the content.

Henri Lefèbvre

\section{Introducción}

Dentro de los estudios latinoamericanos marxistas de la cultura y los medios, planteados en esta misma revista Telos, Alfaro-Vargas (2017), se establece como objeto de estudio, entre otros, las narrativas innaturales y la ciencia ficción (CF). Por tal motivo, analizaremos el caso de estos fenómenos literarios y de masas en el ámbito de su desarrollo en Costa Rica. El caso costarricense es sintomático de estas nuevas formas culturales de neocolonialismo, que se repiten, creemos, a lo largo de Latinoamérica, buscando solamente introducir valores ligados al neoliberalismo y a un capitalismo en crisis.

La CF, por otra parte, se ha convertido en los últimos años en un importante tema de discusión y difusión a nivel mundial. En Costa Rica, desde el 2014, se ha dado una importante reflexión sobre este fenómeno literario tanto en términos filológicos, como sociológicos, vislumbrándose algunos elementos ideológicos caracterizados por una unión público-privada que respalda el surgimiento de este fenómeno. El análisis hasta hoy ha permitido señalar la relación entre la producción de esta CF y el neoliberalismo, en el ámbito no solo costarricense sino también a nivel internacional, en función del denominado novum tecnocrático (Alfaro-Vargas, 2015).

Así, la CF del novum tecnocrático se define, a la vez, como un proceso para mejorar la efectivización de la plusvalía y un medio de control social (Alfaro-Vargas, 
2014b y 2016a). No obstante, el novum tecnocrático conlleva la fantasización de la CF (una insalvable contradicción lógica) (Alfaro-Vargas, 2015), que nos ha llevado a plantear, en este artículo, una redefinición de esta, en el ámbito costarricense, como, más bien, una implementación de la agenda de las narrativas innaturales, lo cual permite ligar el fenómeno, como veremos, al post-postmodernismo y clasificar el grupo de escritores costarricenses de "CF", como la manifestación de la segunda fase de la Promoción de los 80 , en cuanto estos responden a los imperativos derivados de la crisis económica del 2008, con una visión más abiertamente neoliberal e, incluso, con tintes fascistas en algunos casos. Nuestro enfoque se mueve entre lo literario y lo sociológico, como una Literaturwissenschaft ${ }^{1}$.

El fenómeno de la CF en Costa Rica ha dividido las opiniones, en la medida que la derecha académica ha tratado de legitimar tal producción literaria. De hecho, la Escuela de Filología, Lingüística y Literatura de la Universidad de Costa Rica ${ }^{2}$, había ya organizado unas jornadas sobre literatura fantástica y géneros afines (entre ellos, la CF) entre el 18 y 19 de setiembre del 2013, de donde resultaron varias publicaciones, como aquellas de: Ruiz-Ulloa (2014), Soto-Bogantes (2014) y Víquez-Jiménez (2014), cuyas propuestas adolecen de profundidad, a falta de un adecuado manejo teóricopoético de la CF. Asimismo, se debe señalar que el volumen 40, número 1, de la Revista de Filología y Lingüística que recoge tales artículos, también incorpora el texto de Alfaro-Vargas (2014a), escrito (por invitación) desde una perspectiva crítico-marxista, el cual desató una discusión iniciada con la participación de Molina-Jiménez (2014) y Alfaro-Vargas (2014c), extendiéndose esta desde finales del 2014 hasta mediados del 2015.

La tesis que nos guiará será la siguiente: el proceso de fantasización experimentado por la CF conlleva, en el contexto costarricense, que tal producción

\footnotetext{
${ }^{1} \mathrm{Al}$ respecto de este enfoque sociológico de la literatura, véase: (Alfaro-Vargas, 2005a, 2005b y 2007.

${ }^{2}$ De hecho, dentro de la división de funciones existentes alrededor de la denominada CF costarricense, la Universidad de Costa Rica se ha enfocado en la legitimación ideológica de este fenómeno, mientras la Universidad Estatal a Distancia se ha centrado a la publicación de los cuentos ligados a esta corriente literaria.
} 
derive en una manifestación de las narrativas innaturales, lo cual implica que el fenómeno de la $\mathrm{CF}$ en Costa Rica está ligado a las políticas neoliberales postpostmodernas. Así, primeramente, analizaremos la propuesta teórica de Alvarado-Vega (2015), en el ámbito costarricense, como medio para acceder a una discusión sobre los conceptos de CF y fantasía. Seguidamente, será definida la CF en relación con las categorías dialécticas de lo real y lo posible (de cuño lefebvriano) y, finalmente, se explicarán los elementos que permiten clasificar tal producto literario no como $\mathrm{CF}$, sino como una muestra de las narrativas innaturales, en el contexto de la aparición del postpostmodernismo y de la actual crisis del capitalismo.

\section{La CF de acuerdo con Óscar Gerardo Alvarado Vega}

Alvarado-Vega parte de una distinción entre CF y fantasía, en la cual él concibe la fantasía como "la ruptura del orden establecido y reconocido, para dar lugar a lo inadmisible en el seno de lo cotidiano" (2015, p. 3). Luego, si esto define lo opuesto a la $\mathrm{CF}$, entonces la CF vendría a entenderse como algo admisible en lo cotidiano, sin romper el orden establecido y reconocido; por ende, para Alvarado-Vega, la CF se liga a lo existente, al statu quo.

Mas, Alvarado-Vega señala: "el texto de ciencia ficción debe ser verosímil, aunque lo relatado no sea posible" (2015, p. 10), con lo cual, para él, la CF no tiene que "convertirse en un calco de la realidad" $(2015$, p. 10). El texto de CF para este autor más que ligarse a una concepción mimética (realista, referencial) de la realidad, se liga a una concepción no-mimética, es decir, semiótica, en donde el conjunto de signos crea "un mundo que no existe, pero [que] resulta probable y aceptable" (Alvarado-Vega, 2015, p. 10). En otras palabras, para Alvarado-Vega, el texto de CF es areferencial.

Sin embargo, Alvarado-Vega indica: "la ciencia ficción no es ajena a los grandes problemas que afronta la humanidad o a las grandes preocupaciones que enfrenta el ser humano" (2015, p. 15). Por consiguiente, de acuerdo con esta posición, la CF refiere a lo existente, de manera que "no se pierde conexión con lo actual, ni con 
las inquietudes que se afrontan hoy" (2015, p. 15). Luego, hay mímesis y la propuesta de este autor hasta aquí es contradictoria y confusa.

La confusión que se ha evidenciado (dentro de la propuesta de Alvarado-Vega, en la relación entre CF y mímesis) responde al hecho señalado arriba de la falta de precisión a la hora de establecer un adecuado y lógico concepto de CF, porque si este sitúa la CF en el ámbito de lo admisible, ¿cuáles argumentos se ofrecen para explicar: "los viajes en el tiempo, los mutantes, los encuentros con extraterrestres, los superhombres y los universos paralelos" (2015, p. 19) dentro de lo cotidiano y en función del orden establecido y reconocido? Es claro que en su propuesta existe no solo una confusión entre CF y fantasía, sino que también la cuestión de la mímesis dentro de la $\mathrm{CF}$ es abordada de manera problemática por tal autor.

Estos problemas se incrementan cuando Alvarado-Vega refiere a la relación entre $\mathrm{CF}$, ciencia y tecnología. $\mathrm{Al}$ respecto, este autor expresa que: "[1]a ciencia y la tecnología acompañan al ser humano y se convierte en tema de la escritura" (2015, p. 4). Para él, los avances científicos y la tecnología son "lo que le da presencia" (2015, p. 4) a la CF. No obstante, a pesar de que él mismo le otorga correctamente un valor fundamental a la ciencia y la tecnología dentro de la $\mathrm{CF}$, termina afirmando que hay dos tipos de CF: "[1]a que no enfatiza en lo científico y la que sí lo hace” (2015, p. 5), con lo que se hace difícil distinguir qué es CF y qué no lo es, por cuanto si se elimina un elemento fundamental (como lo es lo científico-tecnológico, dentro de la $\mathrm{CF}$ ) no queda entonces ningún elemento discriminante que permita asumir, con una verdadera propiedad conceptual, los rasgos característicos del género de CF: todo podría pasar por CF. Y esto es más evidente, cuando la cuestión del tiempo se desconecta, en la CF, del futuro. A este respecto afirma Alvarado-Vega:

[L]a ciencia ficción no depende de la idea temporal del futuro y su desarrollo en ese momento para ser considerada como tal, pues puede desarrollarse en el presente o el pasado (la idea de pasado, claro está) si tiene el sostén científico [tecnológico] que esta requiere (2015, p. 14). 
Sin una temporalidad futura ${ }^{3}$ y sin tecnología, se hace imposible distinguir la CF de otros géneros.

En síntesis, hasta aquí la posición de Alvarado-Vega nos deja con una concepción de CF que viola el principio de tercero excluido de la lógica formal, el cual reza que algo es $A$ o no- $A$, nunca ambos a la vez. En el caso de la propuesta de este autor, la CF sería mimética y no-mimética, científico-tecnológica y no-científicotecnológica a la vez, o sea, CF y fantasía a la vez, lo cual es insostenible.

Por ahora, es preciso retomar el análisis de la relación entre CF y tiempo, para apuntar algunos problemas ligados con la idea de Alvarado-Vega de una CF elaborada en cualquiera de los tres tiempos: pasado, presente y futuro.

\section{¿Qué es la CF?}

Según Jameson (2005), la fantasía ha sido la beneficiada en su competencia con la CF. Pero esta afirmación implica poder diferenciar entre CF y fantasía.

La fantasía está supeditada al reino de lo irreal, esta no concuerda con las propiedades físicas y racionales de la realidad. Son elementos fantásticos tanto un hada, como un universo paralelo anclado en la teoría de cuerdas.

En términos filosóficos, la fantasía no tiene base onto-epistemológica directa, o sea, es la negación (Negation) $)^{4}$ de lo real, su conexión con la realidad no se da por vía directa y referencial, sino por una discontinuidad que niega lo real material, lo concreto: es un mecanismo de evasión.

\footnotetext{
${ }^{3}$ Sobre la cuestión de la temporalidad, volveremos más adelante y con más profundidad. Se puede también encontrar una explicación de la cuestión temporal en: (Alfaro-Vargas, 2014b).

${ }^{4}$ Esta negación es diferente de la negación implícita, por ejemplo, en el concepto de Aufhebung, ya que tal negación (Negation) conlleva un sesgo de nulidad y discontinuidad totalmente ajeno a la Aufhebung. Al contrario de la Aufhebung, la Negation es la separación del Ser y Deber Ser. La palabra alemana Aufhebung, que hemos traducido como superación, y el verbo aufheben (superar) son realmente intraducibles al español, porque refieren a procesos donde se elimina algo conservándolo; a saber, se pasa de un estado (de una identidad) a otro estado (como diferencia) que ya estaba contenido en el primer estado, pero como posibilidad de desarrollo. La Aufhebung introduce la negatividad en el Ser (es decir, lo posible, no su negación), esta es congruente ( $\mathrm{A} \equiv \mathrm{A})$. Véase al respecto de la noción de Aufhebung: (Hegel, 1976)
} 
En cuanto mecanismo de evasión, la fantasía se funda sobre la violación del tercero excluido de la lógica formal (como veremos, más adelante, asimismo con las narrativas innaturales), en la medida que es y no-es a la vez, la fantasía es un discurso (una forma) que existe y el cual solo es posible dentro de unas condiciones reales y sociales de surgimiento (el contenido real), pero la fantasía no-es en tanto se pretende totalmente alejada y desconectada de tal realidad, es decir, se pretende irreal pero producto de la realidad o, visto de otro modo, es real como forma discursivo-estética pero irreal como contenido pretendidamente arreferencial y a-social, lo cual no deja de ser contradictorio y anulante, porque toda forma discursiva es social y, por ende, tiene un contenido dado por la praxis de la sociedad donde se ampara tal discurso.

Al pretenderse separada de la realidad, la fantasía es discontinua y abstraída, no está enlazada a lo real, no lo continúa; no es concreta y, en consecuencia, ni probable ni posible.

Por otra parte, la CF, a diferencia de la fantasía que pretende desconocer la realidad, asume la representación (lo abstracto) de la realidad (lo concreto) en términos cognitivos, mediante las categorías de lo real y lo posible. En la CF, lo real (el contexto de producción/escritura, donde se origina el texto de $\mathrm{CF}$ ) se transforma en la elaboración estética, con el fin de representar una sociedad posible, que es producto de la transducción ${ }^{5}$ operada sobre lo real mismo. Lo que se efectúa entonces es una extrapolación de las posibilidades de desarrollo ocultas en el contexto de producción/escritura, para mostrar críticamente posibles líneas de desarrollo y de transformación social. La CF es tan política como cualquier otra expresión cultural, pero con una conciencia clara de ello.

Con respecto a la fantasía, la CF conlleva algunos cambios en el trasfondo filosófico que sostiene la fantasía. Si bien los mundos fantásticos se articulan como pretendidas realidades en sí, como discontinuidades adyacentes al mundo real, como

\footnotetext{
${ }^{5}$ Sobre el concepto de transducción, véase: (Lefebvre, 1976).
} 
manifestaciones identitarias $(A=A)$, lo cierto es que la $C F$ se levanta sobre una estructura ontológica no solo móvil, sino también transformable (de ahí su carácter abiertamente político).

El Ser en la CF no se concibe como sustancia, como esencia eterna e inmutable, sino como unidad de Ser y Deber Ser, donde el principio lógico de identidad $(A=A)$ es superado (aufgehoben) por el principio de congruencia $(A \equiv A)$, el cual permite no solo representar estéticamente mundos discontinuos pretendiéndose realidades ontológicas alternas en sí, sino que también representa mundos que -en tanto resultado de la extrapolación de las posibilidades que yacen en lo real mismo- son cognitiva y políticamente la continuidad de lo $\mathrm{real}^{6}$. Es decir, la CF rescata la identidad (dada en la continuidad) y la diferencia (dada en la discontinuidad). El principio de identidad conlleva fijeza, y el de congruencia fijeza y cambio. La congruencia es dialéctica.

La CF funciona sobre un proceso de superación (Aufhebung), donde el Ser es movimiento histórico, social, político y económico, a través de la introducción de la ciencia y la tecnología, dentro del proceso diegético, mediante una mímesis cognitiva cuya finalidad es una acción política específica.

En este marco, la CF requiere, como elemento fundamental, una diégesis asentada en el futuro, en tanto el planteamiento de una tecnología superior a la del contexto de producción/escritura no puede permitirse coincidir con este contexto, sin olvidar el efecto crítico de la CF dado a partir del principio de congruencia que lo sustenta y de la superación (Aufhebung) del contexto de producción/escritura que esto supone.

Si tomamos, por ejemplo, la película The Island (Bay, 2005), vemos que el elemento tecnológico presente (la clonación con fines, en principio, médicos) coincide con el contexto de producción de la película misma, en función del elemento temporal.

\footnotetext{
${ }^{6}$ Lo real no es aquí, en el campo de la CF, un pretexto de modelo alguno como creía Baudrillard (1991), sino que lo real siempre es más rico y complejo que cualquier modelo. Lo real supera (aufhebt) cualquier modelo posible.
} 
Los clones viven en un mundo donde la protagonista es en realidad la doble de una famosa actriz (la misma Scarlette Johansson, en la película), a la cual su clon sirve como reserva de órganos. En la película, la tecnología "futurista" existe ya en el 2005 (tiempo del contexto de producción de esta película, que es compartido con el tiempo de la diégesis de esta), pero como algo clandestino, casi como una conspiración corporativa (muy del gusto del público estadounidense). The Island sería, por ende, CF anclada en el presente y no en el futuro.

No obstante, The Island reduce su crítica a un elemento moralista, ocultando el factor económico que mueve la cuestión de la clonación en la película y el marco capitalista de esto. En consecuencia, no hay crítica, porque el contexto de producción no se inserta, como totalidad, en el juego de las categorías de lo real y lo posible, o sea, en The Island lo posible se subsume y se anula en lo real. En términos lógico/ontoepistemológicos $A$ sigue siendo igual a $A(A=A)$. Por lo general, la $\mathrm{CF}$ anclada en el presente es acrítica y conservadora, políticamente hablando, ya que asumir una crítica fuerte y directa es difícil, en cuanto el sistema tiende a evitar la disidencia política.

Cuando, por otra parte, la CF se pretende anclada en el pasado (con respecto al contexto de producción y escritura), como en la novela española El mapa del tiempo (Palma, 2008), se cae en una serie de situaciones irreales e inverosímiles, como el empleo de nociones venidas de la teoría de cuerdas, en un momento histórico (dentro de la diégesis) donde no existía la propuesta como tal. Aquí no hay crítica, solo evasión del contexto de escritura, en una España marcada en los últimos tiempos por un deterioro de las libertades civiles y, a nivel económico, por la aplicación de una serie de plutocráticas políticas proteccionistas del capital. El mapa del tiempo es realmente fantasía. La CF anclada en el pasado no es CF. El empleo anacrónico de elementos científico-tecnológicos crea una contradicción insalvable, en la aplicación de las categorías de lo real y lo posible.

La película Elysium (Blomkamp, 2013) plantea mayoritariamente una tecnología futurista plausible, donde existe una estación espacial albergando a la clase 
alta, con todos los lujos y comodidades, a la vez que las clases trabajadoras viven en la superficie terrestre totalmente contaminada, generando más y más plusvalía para el bienestar de las clases altas. La crítica a la explotación capitalista y la representación de los medios para superar tal flagelo crean un desfase propio de la aplicación de las categorías de lo real y lo posible, que busca llevar a la reflexión-acción (en términos políticos y de transformación) al espectador. La tecnología futurista permite crear un efecto de extrañamiento (Verfremdungseffekt), el cual busca crear asombro y preocupación, distanciando al espectador de su realidad familiar (Bloch, 1970), "para agudizar la mirada crítica del público y para sensibilizarlo sobre los acontecimientos políticos y sociales" (Spiegel, 2006, p. 15), con el fin de que el espectador pueda ver su propia realidad, a partir de una representación estética que concibe una diégesis que mimetiza el problema que lo aqueja (como clase trabajadora), en el contexto de producción que comparte con la película. Aquí funciona el principio de congruencia $(A \equiv A)$, en tanto se supera (aufhebt) lo real mediante lo posible.

\section{¿CF o fantasía en Costa Rica?}

Volviendo al contexto costarricense, en este apartado, se va a tener como trasfondo los análisis de Alfaro-Vargas (2013 y 2014a), donde se demuestra que la denominada CF costarricense (por lo menos, hasta ese momento) no es tal, con el fin de centrarnos en algunos productos literarios no abordados por este autor, en tales análisis.

Aquí, el análisis girará alrededor de la antología titulada Te voy a recordar. Relatos de ciencia ficción, que contiene los siguientes cuentos: "Pronóstico del tiempo" (Clark, 2015), "Te voy a recordar" (Garro, 2015), "Los turistas siempre fastidian" (González-Chaves, 2015), "Un joven demonio entre las sombras" (Molina-Jiménez, 2015a), "Hacker y el expediente CLO-B=X" (Pérez-Yglesias, 2015), "La máquina de la memoria" (Quesada, 2015), "Círculo perfecto" (Quijano-Vincenzi, 2015) y "La 
incompleta" (Rossi, 2015). Asimismo, les recuerdo, se reflexionará sobre Señora del tiempo (Quijano-Vincenzi, 2014) y Deus ex machina (Garro, 2009).

El relato "Pronóstico del tiempo" establece la temática del viaje en el tiempo, al igual que "Círculo perfecto" de Quijano-Vincenzi, como elemento científicotecnológico no correspondiente a la $\mathrm{CF}$, en tanto la idea de viajar en el tiempo viola el segundo principio de la termodinámica (el principio de entropía), el cual implica que el tiempo tiene una direccionalidad determinada (la flecha del tiempo), yendo desde el pasado hasta el futuro; por ende, no se puede viajar en contra de tal direccionalidad. Luego, estos cuentos son fantasía y no CF.

De Quesada, "La máquina de la memoria" no tiene elementos tecnológicos ni críticos propios de la CF y, más bien, al situar su diégesis en el pasado con respecto al contexto de escritura del autor del cuento, se viola el principio temporal futurista de la $\mathrm{CF}$; en consecuencia, este cuento no es CF.

"Los turistas siempre fastidian", a nivel tecnológico, no introduce nada novedoso y se conforma con recurrir al discurso sobre extraterrestres, para afirmarse como CF, pero sin salirse del contexto de escritura del autor, en cuanto la tecnología se reduce a cosas ya existentes: iPods y televisores a color. Este cuento tampoco es CF.

"Te voy a recordar" se afinca en un recurso poco válido en sí mismo, para ofrecerse como un texto futurista, o sea, el fecharse en el futuro (el 2054 para ser precisos), pero sin crear un contexto diegético futurista. Se habla de computadoras y de clonación, que ya hoy existen. Para solventar este escaso manejo tecnológico-futurista se habla de platillos voladores, pero sin sustento físico o tecnológico, el cual permita creer (verosimilizar) que una nave con esa forma pueda volar en nuestra atmósfera (hay violación de leyes físicas); por ende, este cuento no es CF.

"La incompleta" de Rossi presenta un mundo post-apocalíptico, marcado por una tecnología ya existente en el contexto de escritura de la autora, donde además el espacio diegético es distópico, en cuanto la protagonista (Lalia) se ve sumida finalmente 
en la desesperanza, en una situación sin salida. El final del relato caracteriza este texto como un cuento fatalista, alejado de la CF.

Igualmente, distópico es "Hacker y el expediente CLO-B=X" de PérezYglesias, el cual presenta un informatizado mundo fantástico, donde el sujeto se anula, en función de una alienación cibernética sin salida, dentro de un esquema cibernético del giro lingüístico. Este texto no es CF.

"Un joven demonio entre las sombras" de Molina-Jiménez es un cuento que no se elabora dentro de una diégesis con tecnología futurista, ni con una crítica del establishment y, al contrario, se asienta dentro de una ideología fascista. Así, este cuento presenta a dos sicarios del Primer Mundo, que matan a un disidente (tercermundista), quien protesta contra los intereses de algunas empresas transnacionales. Este cuento afirma como valor el derecho a matar a quien se oponga al capital ${ }^{7}$. Este cuento no es CF.

A modo de paréntesis, permítasenos expresar algunas palabras sobre todos estos cuentos recopilados en el libro Te voy a recordar. Relatos de ciencia ficción, en tanto Molina-Gavilán (2015) afirma que estos cuentos responden al concepto suviniano de novum, pero por su carencia de crítica social y de manejo científico-tecnológico, esto es completamente falso. En estos cuentos, no se da un extrañamiento cognitivo en términos del novum, tal y como Darko Suvin (1979) lo entendió.

Dentro del texto Deus ex machina (Garro, 2009), tenemos la novela corta "Objetivo madre", que presenta una aventura espacial, plagada de una tecnología que responde más bien a los imperativos de la fantasía que a los de la CF. Incluso, se representan habilidades como la telequinesis, la cual también responde a la fantasía. Cuestiones como la capacidad de la nave Tauro 2, para convertirse casi en un rayo

\footnotetext{
${ }^{7}$ Es difícil entender cómo una universidad con principios humanistas (la Universidad Estatal a Distancia) publica un cuento con una ideología de este tipo, que atenta contra el legítimo derecho de los pueblos a la protesta. El derecho a la protesta es un derecho fundamental del ser humano, el cual no puede ser violentado por los intereses de las transnacionales y de los países del Primer Mundo.
} 
luminoso, por la velocidad que alcanza, rompe cualquier vestigio verosimilizante ofrecido por la ciencia y la tecnología. De igual manera, este texto, que fue premiado dentro del Certamen Latinoamericano de Ciencia Ficción, tiene una problemática intertextualidad con la película Sphere (Levinson, 1998), debido a que hay muchos (quizás demasiados) elementos en común. “Objetivo madre” no es CF.

Por otra parte, el otro texto que conforma Deus ex machina, "El niño mariposa" es una secuela de "Objetivo madre", que no aporta nada en términos de CF.

Señora del tiempo (Quijano-Vincenzi, 2015) es una novela cuya diégesis se sostiene en los poderes paranormales de una mujer y en una esfera (de la cual no se dan detalles de su funcionamiento, en términos científico-tecnológicos) donde ambos elementos permiten predecir terremotos. La diégesis de esta novela se ubica en el 2062, pero tecnológicamente no muestra diferencias tecnológicas (amparadas científicamente) con el contexto de escritura de la autora. Señora del tiempo no es CF.

Si como vemos todos estos textos que hemos mencionado de la literatura costarricense, sumados a los analizados por Alfaro-Vargas (2013 y 2014a), entonces es claro que no hay una producción de CF en Costa Rica. No obstante, todos estos textos (los analizados aquí y los de los artículos anteriormente mencionados de Alfaro-Vargas) pueden ser reclasificados en lo que hoy se denomina las narrativas innaturales y ligados al proyecto ideológico-político del neoliberalismo.

\section{Literatura costarricense, Promoción del 80 y las narrativas innaturales}

Dentro del desarrollo de la literatura costarricense, Quesada-Soto (2000) hablaba de la Promoción del 80, donde se agrupa a una serie de autores marcados, entre otras cosas, por dos elementos: el neoliberalismo y el postmodernismo.

Con vistas en esta clasificación de Quesada-Soto, se va aquí a diferenciar entre una primera fase de esta promoción, que responde al auge del neoliberalismo y de la ideología postmoderna en Costa Rica, en un momento donde la caída del Muro de Berlín 
y el fin del bloque soviético, daban la sensación de un capitalismo eterno, ilusión que terminó con el estallido de la crisis financiera en el 2007-2008.

La segunda fase de la Promoción del 80 es la ligada a la etiqueta de CF y relacionada con el escepticismo que produjo la crisis financiera a partir de la primera década del siglo XXI. Esta segunda fase reproduce varios elementos: el ideario de un neoliberalismo con abiertos tintes fascistas y el post-postmodernismo, que es una exacerbación del postmodernismo (Nealon, 2012), sin olvidar su anclaje en lo emocional y en las narrativas innaturales ${ }^{8}$.

En este contexto, el post-postmodernismo es una ideología que acepta que los postmodernos eran ya neoliberales, así como también los post-postmodernos lo son, en relación con la necesidad (dada por la crisis que ha estado afectando al capitalismo) de generar la circulación-reproducción (producción y consumo) de mercancías (Nealon, 2012). El neoliberalismo de este período no es ya una ideología de un capitalismo que se pretendió eterno, sino el de un capitalismo con problemas serios de descenso en la tasa global de ganancia (Harvey, 2010), o sea, el de un capitalismo en quiebra, en una contracción económica, al parecer irreversible. De hecho, en el neoliberalismo, actual, las clases capitalistas intentan "orientar las tendencias inherentes a la dinámica de las fuerzas productivas, en direcciones compatibles con la sobrevivencia de su propia hegemonía" (Duménil y Lévy, 2013, p. 194).

En esta crisis del capitalismo y del neoliberalismo mismo, aparece la dictadura de la emoción cuya función es, según Han, "incrementar la productividad y el rendimiento" (2014, p. 71), en tanto lo emocional permite el despliegue y la expresividad de la identidad y el auto-conocimiento personales (García-Martínez y González, 2016). El giro emocional es una manifestación social que "ha requerido cambios epistemológicos" (García-Martínez y González, 2016, p. 15), ligados de hecho

\footnotetext{
${ }^{8}$ El lector puede acceder a otra visión historiográfica diferente a la planteada aquí, pero que no toma en cuenta los cambios en el escenario económico actual. Véase al respecto: (Pacheco-Solórzano, 2014)
} 
a las tendencias fenomenológicas heredadas del postmodernismo, las cuales se centran en una emocionalidad, en una vivencialidad, que se experienciaría (jerga fenomenológica) "paralela al sentimiento de libertad, al libre despliegue de la personalidad" (Han, 2014, p. 71), en el marco de una visión consumista.

La dictadura de la emoción entonces se funda en un irracionalismo, que percibe la racionalidad, la razón, "como coacción, como obstáculo" (Han, 2014, p. 71), y privilegia los elementos irreflexivos del consumismo, en el cual "se venden significados y emociones" (Han, 2014, p. 70). Y es en este marco que surgen las narrativas innaturales, fundadas en lo irracional.

Así, las narrativas innaturales", que "han ganado una tremenda influencia en los últimos años” (Nielsen, 2011, p. 57), juegan un rol importante, en relación con la dictadura de la emoción, donde el término innatural refiere "a representaciones de lo imposible" (Alber, 2016, p. 15). Esta imposibilidad que caracteriza las narrativas innaturales es irracional, debido a la elaboración de "escenarios y eventos física, lógica y humanamente imposibles" (Alber, 2016, p. 14), lo cual sería supuestamente un rompimiento no solo con la mímesis/referencialidad del lenguaje, sino que también la violación del sentido común (McHale, 2012). Las narrativas innaturales afirman que el realismo literario oculta la artificiosidad del texto, mientras ellas hacen gala de tal característica (Richardson, 2013), o sea, hay en estas narrativas innaturales "una motivación anti-mimética, contra-realista o escéptica" (Berry, 2012, p. 129). Las narrativas innaturales lucharían contra el reduccionismo mimético (Alber y otros, 2010). Así, las hadas, los viajes en el tiempo, los multiversos, etc., son cuestiones irreales que, a pesar de poder representarse, por ejemplo, literaria o fílmicamente, no tienen ni pueden llegar a tener existencia.

De hecho, las narrativas innaturales son irracionales y, en consecuencia, irreales, al poner el acento en lo emocional (Alfaro-Vargas, 2017). Por ejemplo, si

\footnotetext{
${ }^{9}$ El lector puede profundizar en la crítica de los fundamentos teóricos y epistemológicos (la teoría de conjuntos) de las narrativas innaturales, en: (Alfaro-Vargas, 2016b).
} 
recordamos la película Interstellar (Nolan, 2014), podremos ver que en su trama se dice que la única fuerza capaz de atravesar un agujero de gusano es la gravedad (que es una fuerza física). No obstante, a medida que se desarrolla la trama y el anclaje científico que pretendía fundamentar su diégesis pierde validez; entonces se dice que el amor también puede atravesar el agujero de gusano y así acceder a otras dimensiones. De esta manera, la emoción (el amor) de forma sumamente irracional, se inserta en una problemática física (ya cuestionable como lo es la teoría de cuerdas) como elemento innatural implicando que el amor es a la vez una emoción y una fuerza física semejante a la gravedad. Luego, aquí se marca un elemento fundamental en gran parte de la dictadura de la emoción y de las narrativas innaturales, o sea, la violación del principio lógico de tercero excluido de la lógica formal (al igual que la fantasía lo hace), de manera que un relato (fílmico, literario, etc.) es construido con una estructura, en la cual " $p$ y no- $p$ son simultáneamente verdaderos" (Alber y otros, 2013, p. 105) y donde hay una transformación de estos "mundos imaginados en franquicias comercialmente exitosas (Star Wars, Lord of the Rings, etc.)" (Hatavara y otros, 2016, p. 3).

Dentro de las narrativas innaturales, el negocio es completo, pues se efectiviza plusvalía (elemento ligado al neoliberalismo en crisis y al post-postmodernismo) y se "estupidizan" las masas con la socialización de patrones irracionales, que se pasan por elementos cognitivos, por realidades alternas, en tanto la posible situación planteada dentro de productos culturales (cine, literatura, etc.) en consonancia con las narrativas innaturales, “está necesariamente ligada al 'aquí y ahora' del receptor" (Zeman, 2016, p. 19), así como a un proceso donde se pretende eliminar el proceso lógico (de ahí la anulación del tercero excluido de la lógica formal y, en consecuencia, del principio de contradicción de la misma lógica) para dejar espacio solo para una "aprehensión" emocional, a-lógica, vivencial, fenomenológica. Claramente, en este momento histórico, la erróneamente denominada $\mathrm{CF}$, pero que realmente es manifestación de lo fantástico o de lo innatural, "es usada como un medio para el adoctrinamiento" (May, 2017, p. x). 
Las narrativas innaturales, en el actual contexto, responden a una estrategia post-postmoderna, la cual lleva a estas prácticas culturales (en tanto textos literarios y en cuanto reflexión teórico-narratológica) a centrarse en "los poderes de lo falso de la literatura, sus habilidades post-postmodernas de crear otros, virtuales, mundos" (Nealon, 2012, p.158; énfasis mío). Es decir, hoy las narrativas innaturales se establecen como un paradigma narratológico, donde "lo que cuenta como ficción es aquello que las instituciones dominantes llaman ficción" (Stock, 2016, p. 211). En otras palabras, lo que cuenta como ficción es lo que el proyecto post-postmoderno (neoliberal) define como tal. Ya Marx y Engels afirmaban: "Las ideas dominantes de una época siempre fueron solamente las ideas de la clase dominante" (Die herrschenden Ideen einer Zeit waren stets nur die Ideen der herrschenden Klasse) (1977, p. 480) y, en este momento, las narrativas innaturales juegan ese rol.

Dentro del ámbito del giro emocional del post-postmodernismo y de las narrativas innaturales, los textos de la segunda fase de la Promoción del 80, ligada a la equívoca etiqueta de $\mathrm{CF}$, se reclasifican como manifestaciones de las narrativas innaturales en la literatura costarricense, al igual que el texto de Ortega-Rodríguez y Víquez-Jiménez (2015) - Los peces de Cooper ${ }^{10}$ - que también se inserta en esta línea no desde la CF, sino desde el género detectivesco (Alfaro-Vargas, 2016c). Asimismo, en lo que se ha denominado mercantilmente como CF costarricense, podemos ver lo innatural en ideas como: el motor de plasma (Garro, 2009), el contacto transtemporal (Clark, 2015), los platillos voladores o naves espaciales (Garro, 2015 y GonzálezChaves, 2015), la teletransportación y la capacidad de predecir el futuro (QuijanoVincenzi, 2014 y 2015), la reproducción humana por partenogénesis (Rossi, 2015), todos ejemplos válidos de ello.

\footnotetext{
${ }^{10}$ Esta novela se inscribe dentro de las narrativas naturales no por su ligamen a la $\mathrm{CF}$, sino por representar una ontología alterna donde es posible producir electricidad, mediante superconductores que funcionan a temperatura ambiente, lo cual es imposible en nuestras condiciones físicas. A diferencia de los demás textos literarios analizados aquí, esta novela experimental es de buena calidad.
} 
De hecho, para Alber (2009), lo innatural tiene grados que se pueden medir de acuerdo con la desviación que se tenga en cada texto anclado en las narrativas innaturales, con respecto a las estructuras del mundo real. Por eso, entre la literatura costarricense de corte innatural podemos ver diferentes niveles de elaboración textual ligados a tales narrativas, pero siempre con su trasfondo neoliberal y post-postmoderno. De igual modo, aplicar la noción de innatural a textos anteriores al contexto de surgimiento de estas narrativas (marcado por la crisis del neoliberalismo) sería un anacronismo, por lo que sería mejor optar por las simples categorías de lo gótico y lo fantástico para textos más antiguos, como lo hace Cubillo-Paniagua (2014) y así evitar errores de clasificación, como el cometido por Molina-Jiménez (2015b), al anacrónicamente definir un texto que no es $\mathrm{CF}$, como tal. Para el caso del cuento presentado por Molina-Jiménez, titulado "El número 13,013" de León Fernández Guardia, es mejor abordarlo desde el planteamiento de Cubillo-Paniagua (2014) y nunca como CF.

De esta manera, autores como: Jessica Clark, Iván Molina Jiménez, Laura Quijano Vincenzi, Evelyn Ugalde, Mariana Castillo Rojas, Manuel Delgado, Antonio Chamu, Luis E. Jara, Daniel Garro, Ericka Lippi Rojas, David Díaz Arias, Laura Casasa Núñez, Alberto Ortiz, José Ricardo Chaves, Daniel González Chaves, Ana Cristina Rossi, Uriel Quesada y María Pérez Yglesias, representan las nuevas narrativas innaturales en la literatura costarricense y no la CF. Ellos son la segunda fase de la Promoción del 80.

\section{Conclusiones}

Las reflexiones de Alvarado-Vega nos han permitido profundizar en las nociones de fantasía y CF. Así, mediante el diálogo planteado aquí se ha podido establecer los criterios para determinar el ámbito conceptual de la fantasía y la $\mathrm{CF}$, no solo de manera teórica, sino también a través de la muestra empírica, en el cine y la literatura. De hecho, el proceso de fantasización dado dentro de la CF implica, en el 
contexto costarricense, que tal producción se define como una manifestación de las narrativas innaturales, lo cual implica que el fenómeno de la CF en Costa Rica está ligado a las políticas neoliberales post-postmodernas.

De igual modo, analizando la producción de varios autores costarricense, se llega a la conclusión de la inexistencia de una literatura costarricense de CF y, más bien, se afirma, a través de la reclasificación de la producción literaria costarricense referida aquí, que en la literatura costarricense lo que se ha evidenciado es el desarrollo de las narrativas innaturales, en su versión de fantasía.

El grupo de autores, mencionado anteriormente, se ha ubicado dentro de la segunda fase de la Promoción del 80, con características ideológicas ligadas a la crisis del neoliberalismo y al post-postmodernismo (y en algunos casos con una abierta tendencia fascista), tratando de generar mercancías que no solo permitan la circulaciónreproducción del capital, sino que también generen un excedente ideológico como medio de control social y de adoctrinamiento.

Asimismo, se ha mostrado el vínculo entre la producción literaria de esta segunda fase de la Promoción del 80 y algunos procesos ligados al giro emocional y a los requerimientos socio-económicos y psicológicos del neoliberalismo actual, que explican el marco de surgimiento de las narrativas innaturales.

Las narrativas innaturales son una estrategia neoliberal de control ideológico y de reproducción-circulación del capital, que se manifiesta como una propuesta narratológica de carácter irracional, debido esto a la eliminación del tercero excluido de la lógica formal, donde estas se fundamentan.

\section{Referencias Bibliográficas}

Alber, Jan. (2009). Impossible Story worlds - and What to Do with Them. Story Worlds: A Journal of Narrative Studies. Volume 1. Estados Unidos. (Pp. 79-96). DOI: $10.1353 /$ stw.0.0008 
Alber, Jan. (2016). Unnatural Narratives. Impossible Worlds in Fiction and Drama. University of Nebraska Press. Estados Unidos y Reino Unido.

Alber, Jan; Iversen, Stefan; Nielsen, Henrik Skov y Richardson, Brian. (2010). Unnatural Narratives, Unnatural Narratology: Beyond Mimetic Models. Narrative. Volume 18, number 2. Estados Unidos. (Pp. 113-136).

Alber, Jan; Iversen, Stefan; Nielsen, Henrik Skov y Richardson, Brian. (2013). What Really Is Unnatural Narratology? Story Worlds: A Journal of Narrative Studies. Volume 5. Estados Unidos. (Pp. 101-118).

Alfaro-Vargas, Roy. (2005a). Relación literatura-sociedad: una aproximación teórica. Revista de Ciencias Sociales. Volumen II, número 108. Costa Rica. (Pp. 7178).

Alfaro-Vargas, Roy. (2005b). Sociología dialéctica de la literatura: cuestión de método.

Revista de Ciencias Sociales. Volumen III-IV, número 109-110. Costa Rica. (Pp. 151-156).

Alfaro-Vargas, Roy. (2007). Forma y movimiento en los estudios literarios. Revista de Ciencias Sociales. Volumen III-IV, número 117-118. Costa Rica. (Pp. 149158).

Alfaro-Vargas, Roy. (2013). La ficción de Iván Molina Jiménez. Letras. Número 53. Costa Rica. (Pp. 201-217).

Alfaro-Vargas, Roy. (2014a). El novum en la ciencia ficción costarricense. Revista de Filología y Lingüística. Volumen 40, número 1. Costa Rica. (Pp. 129-140).

Alfaro-Vargas, Roy. (2014b). Marxismo y ciencia ficción. Praxis. Número 72. Costa Rica. (Pp. 81-97).

Alfaro-Vargas, Roy. (2014c). Pseudo-ciencia ficción costarricense. La Nación. 25 de octubre. Costa Rica. (p. 31A).

Alfaro-Vargas, Roy. (2015). El novum tecnocrático. Hélice. Volumen II, número 5. España. (Pp. 6-20).

Alfaro-Vargas, Roy. (2016a). The Protos Mandate: ciencia ficción y neoliberalismo. Letras. Número 59. Costa Rica. (Pp. 77-97).

Alfaro-Vargas, Roy. (2016b). Las narrativas innaturales. Letras. Número 60. Costa Rica. (Pp. 185-212). DOI: http://dx.doi.org/10.15359/rl.2-60.9

Alfaro-Vargas, Roy. (2016c). Los peces de Cooper y el género detectivesco: del dominante epistemológico al dominante ontológico. Revista Espiga. Volumen XV, número 32. Costa Rica. (Pp. 153-167). 
Alfaro-Vargas, Roy. (2017). Crítica de los estudios marxistas de la cultura y los medios. Telos. Revista de estudios interdisciplinarios en Ciencias Sociales. Volumen 19, número 2. Venezuela. (Pp. 192-211).

Alvarado-Vega, Óscar Gerardo. (2015). La literatura de ciencia ficción: Una mirada al futuro en tiempo presente. Revista Humanidades. Volumen 5, número 2. Costa Rica. (Pp. 1-21). DOI: 10.15517/h.v5i2.21211

Baudrillard, Jean. (1991). Simulacra and Science Fiction. Science Fiction Studies. Volumen 18, número 3. Estados Unidos. (Pp. 309-313).

Bay, Michael. (director). (2005). The Island. DreamWorks Pictures y Warner Bros. Pictures. Estados Unidos.

Berry, R. M. (2012). Metafiction. The Routledge Companion to Experimental Literature. Editorial Routledge. Estados Unidos y Reino Unido.

Bloch, Ernest. (1970). Entfremdung, Verfremdung: Alienation, Estrangement. The Drama Review: TDR. Volume 15, number 1. Estados Unidos. (Pp. 120-125).

Blomkamp, Neill. (director). (2013). Elysium. TriStar Pictures, Sony Pictures Entertainment (SPE), Media Rights Capital, QED International, Simon Kinberg Productions. Estados Unidos.

Clark, Jessica. (2015). Pronóstico del tiempo. Te voy a recordar. Relatos de ciencia ficción. Editorial UNED. Costa Rica.

Cubillo-Paniagua, Ruth. (2014). El surgimiento del relato fantástico en la Costa Rica de la primera mitad del siglo XX. Brumal. Volumen II, número 2. España. (Pp. 161-175). DOI: $\underline{10.5565 / \mathrm{rev} / \mathrm{brumal} .104}$

Duménil, Gérard y Lévy, Dominique. (2013). Neoliberalism and its Crisis. Crises of Global Economies and the Future of Capitalism: Reviving Marxian crisis theory. Editorial Routledge. Estados Unidos.

García-Martínez, Alejandro y González, Ana Marta. (2016). Emotional Culture and TV Narratives. Emotions in Contemporary TV Series. Editorial Palgrave McMillan. Reino Unido y Estados Unidos.

Garro, Daniel. (2009). Deus ex machina. Editorial UNED. Costa Rica.

Garro, Daniel. (2015). Te voy a recordar. Te voy a recordar. Relatos de ciencia ficción. Editorial UNED. Costa Rica.

González-Chaves, Daniel. (2015). Los turistas siempre fastidian. Te voy a recordar. Relatos de ciencia ficción. Editorial UNED. Costa Rica.

Han, Byung-Chul. (2014). Psicopolítica. Neoliberalismo y nuevas técnicas de poder. Editorial Herder. España. 
Harvey, David. (2010). The Enigma of Capital and the Crisis of Capitalism. Editorial Profile Books. Reino Unido.

Hatavara, Mari; Hyvärinen, Matti; Mäkelä, María y Mäyrä, Frans (eds.). (2016). Introduction: Minds in Action, Interpretive Traditions in Interaction. Narrative Theory, Literature, and New Media: Narrative Minds and Virtual Worlds. Editorial Routledge. Reino Unido y Estados Unidos.

Hegel, Georg Wilhelm Friedrich. (1976). Ciencia de la lógica. Editorial Solar y Hachette. Argentina.

Jameson, Fredric. (2005). Archaeologies of the Future. The Desire Called Utopia and Other Science Fictions. Editorial Verso. Reino Unido y Estados Unidos.

Lefebvre, Henri. (1976). Lógica formal, lógica dialéctica. Editorial Siglo Veintiuno. México.

Levinson, Barry. (director). (1998). Sphere. Warner Bros. Pictures. Estados Unidos.

Marx, Karl y Engels, Friedrich. (1977). Manifest der Kommunistischen Partei. Werke Band 4. Editorial Dietz. Alemania.

May, Andrew. (2017). Pseudoscience and Science Fiction. Editorial Springer. Suiza. DOI: $\underline{10.1007 / 978-3-319-42605-1}$

McHale, Brian. (2012). Postmodernism and Experiment. The Routledge Companion to Experimental Literature. Editorial Routledge. Reino Unido y Estados Unidos.

Molina-Gavilán, Yolanda. (2015). Presentación. Te voy a recordar. Relatos de ciencia ficción. Editorial UNED. Costa Rica.

Molina-Jiménez, Iván. (2014). El libre juego de la irracionalidad. La Nación. 22 de octubre. Costa Rica. (p. 31A).

Molina-Jiménez, Iván. (2015a). Un joven demonio entre las sombras. Te voy a recordar. Relatos de ciencia ficción. Editorial UNED. Costa Rica.

Molina-Jiménez, Iván. (2015b). León Fernández Guardia y "El número 13,013”. Alambique: Revista académica de ciencia ficción y fantasía. Volumen 3, número 1. Estados Unidos. (Pp.1-15). DOI: 10.5038/2167-6577.3.1.1

Nealon, Jeffrey T. (2012). Post-postmodernism or, The Cultural Logic of Just-inTime Capitalism. Editorial de la Stanford University. Estados Unidos.

Nielsen, Henrik Skov. (2011). Fictional Voices? Strange Voices? Unnatural Voices? Strange Voices in Narrative Fiction. Editorial De Gruyter. Alemania. 
Nolan, Christopher. (director). (2014). Interstellar. Warner Bros., Syncopy, Paramount Pictures, Legendary Pictures y Lynda Obst Productions. Estados Unidos.

Ortega-Rodríguez, Manuel y Víquez-Jiménez, Alí. (2015). Los peces de Cooper. Editorial UNED. Costa Rica.

Pacheco-Solórzano, Carlos. (2014). Discursos literarios en Costa Rica. Revista Espiga. Volumen XIII, número 27. Costa Rica. (Pp. 59-66).

Palma, Félix J. (2008). El mapa del tiempo. Editorial Algaida. España.

Pérez-Yglesias, María. (2015). Hacker y el expediente CLO-B=X. Te voy a recordar. Relatos de ciencia ficción. Editorial UNED. Costa Rica.

Quesada, Uriel. (2015). La máquina de la memoria. Te voy a recordar. Relatos de ciencia ficción. Editorial UNED. Costa Rica.

Quesada-Soto, Álvaro. (2000). La narrativa costarricense del último tercio de siglo. Letras. Número 32. (Pp. 17-43).

Quijano-Vincenzi, Laura. (2014). Señora del tiempo. Editorial Uruk. Costa Rica.

Quijano-Vincenzi, Laura. (2015). Círculo Perfecto. Te voy a recordar. Relatos de ciencia ficción. Editorial UNED. Costa Rica.

Richardson, Brian. (2013). Unnatural Stories and Sequences. A Poetics of Unnatural Narrative. Editorial de The Ohio State University. Estados Unidos.

Rossi, Anacristina. (2015). La incompleta. Te voy a recordar. Relatos de ciencia ficción. Editorial UNED. Costa Rica.

Ruiz-Ulloa, Geaninni. (2014). Entredichos de la sociedad democrática costarricense: ciencia ficción y política. Revista de Filología y Lingüística. Volumen 40, número 1. Costa Rica. (Pp. 107-112).

Soto-Bogantes, Carlos. (2014). El mundo natural y el futuro de la colonialidad: "Frente frío" de Jessica Clark y "La flor del crepúsculo" de Laura Quijano. Revista de Filología y Lingüística. Volumen 40, número 1. Costa Rica. (Pp. 99-104).

Spiegel, Simon. (2006). Der Begriff der Verfremdung in der Science-Fiction-Theorie. Ein Klärungsversuch. Quarber Merkur. Franz Rottensteiners Literaturzeitschrift für Science Fiction und Phantastik. Números 103/104. Alemania. (Pp. 13-40).

Stock, Kathleen. (2016). Imagination and Fiction. The Routledge Handbook of Philosophy of Imagination. Editorial Routledge. Reino Unido y Estados Unidos. 
Suvin, Darko. (1979). Metamorphoses of Science Fiction. On the Poetics and History of a Literary Genre. Editorial de Yale University Press. Estados Unidos.

Víquez-Jiménez, Alí. (2014). Lectura de dos relatos de ciencia ficción costarricense: "Órdago" y "Objeto no identificado". Revista de Filología y Lingüística. Volumen 40, número 1. Costa Rica. (Pp. 87-95).

Zeman, Sonja. (2016). Perspectivization as a link between narrative micro- and macrostructure. Perspectives on Narrativity and Narrative Perspectivization. Editorial John Benjamins B.V. Holanda y Estados Unidos. 